ESAIM: PROCEEDINGS, August 2010, Vol. 30, p. 70-89

D. Bresch, V. Calvez, E. Grenier, P. Vigneaux \& J.-F. Gerbeau, Editors

\title{
ASYMPTOTIC ANALYSIS OF BLOOD FLOW IN STENTED ARTERIES: TIME DEPENDENCY AND DIRECT SIMULATIONS*,**
}

\author{
Vuk Milišić ${ }^{1}$, Amélie Rambaud ${ }^{2}$ And Kirill Pichon Gostaf ${ }^{3}$
}

\begin{abstract}
This work aims to extend in two distinct directions results recently obtained in [10]. In a first step we focus on the possible extension of our results to the time dependent case. Whereas in the second part some preliminary numerical simulations aim to give orders of magnitudes in terms of numerical costs of direct 3D simulations.

We consider, in the first part, the time dependent rough problem for a simplified heat equation in a straight channel that mimics the axial velocity under an oscillating pressure gradient. We derive first order approximations with respect to $\epsilon$, the size of the roughness. In order to understand the problem and set up correct boundary layer approximations, we perform a time periodic fourier analysis and check that no frequency can interact with the roughness. We show rigorously on this toy problem that the boundary layers remain stationary in time (independent on the frequency number). Finally we perform numerical tests validating our theoretical approach.

In the second part, we determine actual limits, when running three-dimensional blood flow simulations of the non-homogenized stented arteries. We solve the stationary Stokes equations for an artery containing a saccular aneurysm. Consecutive levels of uniform mesh refinement, serve to relate spatial resolution, problem scale, and required computation time. Test computations are presented for femoral side aneurysm, where a simplified ten-wire stent model was placed across the aneurysm throat. We advocate the proposed stent homogenization model, by concluding that an actual computation power is not sufficient to run accurate, direct simulations of a pulsatile flow in stented vessels.
\end{abstract}

\section{INTRODUCTION}

Rupture of aneurysm are common lethal pathologies in western countries. It is mainly due to a loss of elastic properties of tissues that constitutes the arterial walls on some branching. Recently emerged a new kind of stent: a metallic wired mutli-layered prosthesis (see fig. 1 right) that unlike the classical endograft stent need not to be sutured to the arterial wall. Their form-memory metallic structure allow self-expansion recovering the original form without a need of a balloon.

A recent work of the first author establishes, thanks to asymptotic analysis tools, several advantages of this new device [10]. These can be summarized as follows :

\footnotetext{
* The authors would like to thank Cardiatis (www. cardiatis. com), the industrial partner of the Cemracs 09 project Rugosity for the financial support

** We would like to thank also the Institut des systèmes complexes for partial funding of this project as well

1 Wolfgang Pauli Institute (WPI), UMI CNRS 2841,Vienna, AUSTRIA

2 ICJ, UMR 5208, Lyon I

${ }^{3}$ Université Pierre et Marie Curie (UPMC), UMR 7598, Paris, FRANCE
}

(C) EDP Sciences, SMAI 2010 

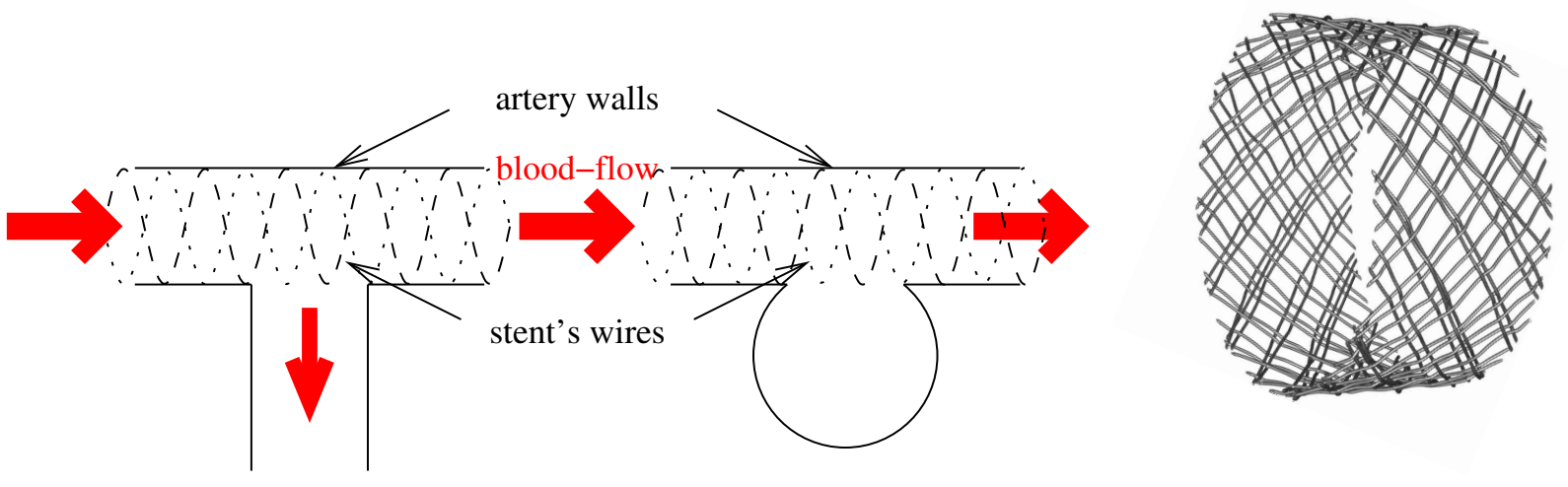

Figure 1. A sketch of stented arteries: with a collateral artery (left), an aneurysmal sac (middle) and a 3D example of a real metallic multi-wired stent (right)

- the presence of a stent at the inlet of a collateral artery (see fig. 1 left) gives rise to a secondary flow explicitly computable : it depends on the pressure jump occurring at zero order (when the stent totally closes the inlet of the collateral artery) and on some periodic microscopic resistivity (computed independently of any kind of macroscopic flow).

- the presence of a stent above a closed aneurysmal sac (see fig. 1 middle) imposes a constant averaged pressure inside the sac, it also inverts the direction of rotation of the vortex running inside the sac: without a stent, the cavity is driven by the mean flow in the artery, the vortex is tangential to the mean flow whereas the pressure jump across the interface imposes an entering velocity profile upward the sac and an outgoing profile downward the middle of the sac.

These results were established theoretically and numerically for the steady Stokes system of equations. In this work, we set up a preliminary toy framework in order to extend those results to the unsteady case.

Although this is not a first attempt to consider the unsteady regime within the boundary layer framework (let's mention $[3,6]$ ), we set up here very basic model for the time periodic case. In the context of blood flow this regime is quite well-suited since the heart delivers a periodic pressure flow impulse to the cardio-vascular system. Another advantage of this work is that it is self-consistent: extending tools presented in [2], we provide self-contained proofs for every step of our approximation process. We give, for instance, a direct proof for time periodic very weak solutions.

The paper is organized as follows: in a first section we give the basic notations and hypotheses of this work, in the next section we construct a boundary layer approximation and then we show that an averaged approximation, cheaper from the computational point of view, is possible. At each step we provide theoretical error estimates wrt the direct rough solution. An interesting feature of the wall-law is exhibited: we show that although we recover the standard $\epsilon^{\frac{3}{2}}$ convergence rate in $L^{2}\left(\Omega_{0}\right)$ norm, the a priori estimates provide only $\epsilon^{\frac{1}{2}}$ rate performing a similar error as the zero order estimate itself. The last section validates numerically theoretical claims stated and proved in previous sections. The poor $H^{1}\left(\Omega_{0}\right)$ error is observed also on the numerical side. In the second part of this paper, we run a series of direct, three-dimensional flow simulations of a femoral artery with a side saccular aneurysm. We examine the relation between discretization parameter, problem scale, and computation time required to solve the stationary Stokes equations. Finite element model of a two-layer 32 wire stent was constructed. We demonstrate that its coarse mesh could not be accurately incorporated in the finest discretization of the blood medium. Finally, a simplified ten-wire stent model was build. The results of the stented versus the unstented vessel show substantial difference in flow pattern inside the aneurysmal pouch. Concluding remarks and possible perspectives are given at the end of the paper. 


\section{The TIME DEPENDENCY}

\subsection{Notations and problem setting}

In this work, $\Omega^{\epsilon}$ denotes the rough domain in $\mathbb{R}^{2}$ depicted in fig. $2, \Omega_{0}$ denotes the smooth one, and $\Omega_{\epsilon} \backslash \Omega_{0}$ the complementary rough sub-domain. $\Gamma_{\epsilon}$ is the rough boundary and $\Gamma_{0}$ (resp. $\Gamma_{1}$ ) the lower (resp. upper) smooth one (see fig. 2).

Hypotheses 1.1. The rough boundary $\Gamma_{\epsilon}$ is described as a periodic repetition at the microscopic scale of a single boundary cell $P_{0}$. The latter can be parametrized as the graph of a Lipschitz function $f:[0,2 \pi[\rightarrow[-1: 0[$ such that

$$
P_{0}=\left\{y \in[0,2 \pi] \times\left[-1: 0\left[\text { s.t. } y_{2}=f\left(y_{1}\right)\right\} .\right.\right.
$$

Moreover, we suppose that $f$ is negative definite, i.e. there exists a positive constant $\delta$ such that $f\left(y_{1}\right)<\delta$ for all $y_{1} \in[0,2 \pi]$. Then the macroscopic boundary $\Gamma_{\epsilon}$ is parametrized as

$$
\Gamma_{\epsilon}=\left\{x \in \mathbb{R}^{2} \text { s.t. } x_{2}=\epsilon f\left(\frac{x}{\epsilon}\right)\right\} \text {. }
$$

We assume that the ratio between $L$ (the width of $\Omega_{0}$ ) and $2 \pi \epsilon$ (the width of the periodic cell) is always an integer called $N$. We consider a simplified setting that avoids the theoretical difficulties and the non-linear
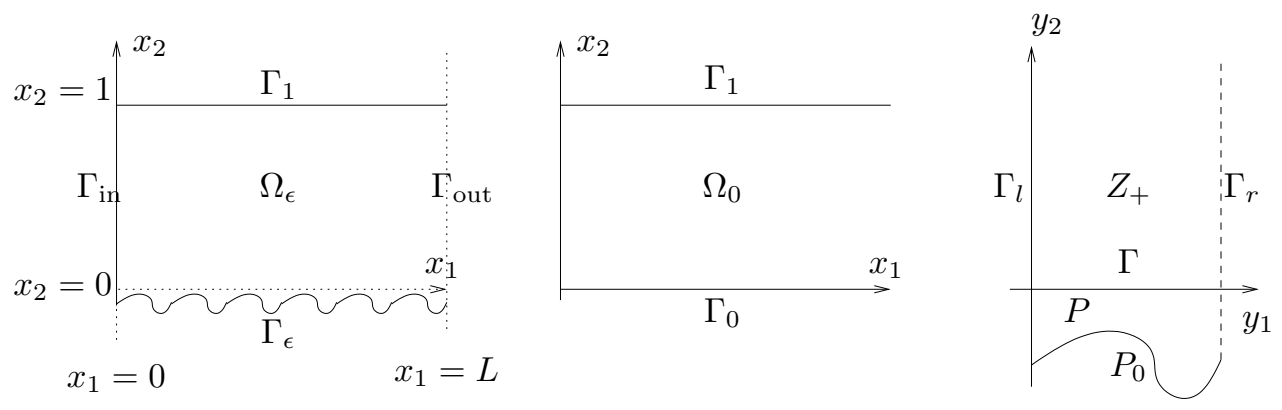

Figure 2. Rough, smooth and cell domains

complications of the full time-dependent Navier-Stokes equations. Starting from the time-dependent Stokes system, we consider a heat-like simplified problem for $u_{\epsilon}$, the axial component of the velocity. The oscillating pressure gradient is assumed to reduce to a time-periodic space-constant right hand side $C(t)$. For sake of conciseness, we consider only periodic inflow and outflow boundary conditions on $u_{\epsilon}$. The simplified problem reads : find $u_{\epsilon}$ such that

$$
\left\{\begin{array}{l}
\partial_{t} u_{\epsilon}-\Delta u_{\epsilon}=C(t), \quad \text { for } x \in \Omega_{\epsilon}, \\
u_{\epsilon}=0, \quad \text { for } x \in \Gamma_{\epsilon} \cup \Gamma_{1}, \\
u_{\epsilon} \text { is } x_{1} \text { periodic. }
\end{array}\right.
$$

We underline that the results below can be directly extended to rough domains with smooth holes and to the Stokes system in the case of a simple sheared flow.

In what follows, functions that do depend on $y=x / \epsilon$ should be indexed by an $\epsilon$ (e.g. $\left.\hat{\mathcal{U}}_{\epsilon, k}=\hat{\mathcal{U}}_{\epsilon, k}(x, x / \epsilon)\right)$. 


\subsection{Time fourier analysis and boundary layer approximations}

Applying the time-fourier transform on (2) one obtains for each frequency-mode $k \in \mathbb{Z}^{*}$ the problem: find $\hat{u}_{\epsilon, k}$ s.t.

$$
\begin{cases}(i k-\Delta) \hat{u}_{\epsilon, k}=\hat{C}_{k} & \text { in } \Omega_{\epsilon}, \\ \hat{u}_{\epsilon, k}=0 & \text { on } \Gamma_{\epsilon} \cup \Gamma_{1}, \\ \hat{u}_{\epsilon, k} x_{1}-\text { periodic on } \Gamma_{\text {in }} \cup \Gamma_{\text {out }} & \text { on } \Gamma_{\text {in }} \cup \Gamma_{\text {out }} .\end{cases}
$$

where $\hat{C}_{k}$ is the fourier mode associated to the frequency $k \in \mathbb{Z}$ :

$$
\hat{C}_{k}:=\frac{1}{2 \pi} \int_{0}^{2 \pi} C(t) e^{i k t} d t, \quad C(t)=\sum_{k} \hat{C}_{k} e^{-i k t}
$$

For the rest of the paper, one denotes $\mathcal{L}_{k}:=(i k-\Delta)$. When $k \equiv 0$ one returns to the steady case already extensively studied in [2], so we only consider $k \in \mathbb{Z}^{*}$ for the rest of this paper.

\subsubsection{The zero order approximation}

Passing to the limit formally wrt $\epsilon$ in (3), one shows rigorously below that actually $\hat{u}_{\epsilon, k}$ converges to $\hat{u}_{0, k}$ solving

$$
\begin{cases}\mathcal{L}_{k} \hat{u}_{0, k}=\hat{C}_{k} & \text { in } \Omega_{0} \\ \hat{u}_{0, k}=0 & \text { on } \Gamma_{0} \cup \Gamma_{1}, \\ \hat{u}_{0, k} x_{1}-\text { periodic on } \Gamma_{\text {in }} \cup \Gamma_{\text {out }} & \text { on } \Gamma_{\text {in }} \cup \Gamma_{\text {out }} .\end{cases}
$$

The solution of this problem is explicit wrt to the data $\hat{C}_{k}$ and the frequency $k$, it reads for every $x \in \Omega_{0}$ :

$$
\hat{u}_{0, k}=\frac{\hat{C}_{k}}{i k}\left(1+A e^{r x_{2}}+B e^{-r x_{2}}\right), \quad r:=\frac{\sqrt{2 k}}{2}(1+i), \quad A:=\frac{e^{-r}-1}{e^{r}-e^{-r}}, \quad B:=\frac{1-e^{r}}{e^{r}-e^{-r}} .
$$

In order to estimate the error made when we consider the solution $\hat{u}_{0, k}$ as an approximation of $\hat{u}_{\epsilon, k}$, we have to extend $\hat{u}_{0, k}$ to the whole rough domain $\Omega_{\epsilon}$. It suffices that it is continuous, since we need $H^{1}$ functions for a priori error estimates. In the literature, either the solution is extended by a constant in the rough layer [7] or one constructs a linear extension using the Taylor expansion around the point $\left(x_{1}, 0\right)$ [1]. In order to correct these errors at the next order, in the first case one corrects then the jump of the derivative, and in the second case one should lift the Dirichlet error [2]. Here, we chose to extend $\hat{u}_{0, k}$ by a linear function in $\Omega_{\epsilon} \backslash \Omega_{0}$ :

$$
\hat{u}_{0, k}:=\left\{\begin{array}{ll}
\hat{u}_{0, k} & \text { in } \Omega_{0}, \\
\mathcal{M}_{k} x_{2} & \text { in } \Omega_{\epsilon} \backslash \Omega_{0},
\end{array}, \text { where } \mathcal{M}_{k}:=\frac{\partial}{\partial x_{2}} \hat{u}_{0, k}\left(x_{1}, 0\right)=\frac{\hat{C}_{k} r\left(2-e^{r}-e^{-r}\right)}{i k\left(e^{-r}-e^{r}\right)} .\right.
$$

\subsubsection{Zero order error estimates}

We detail here the error estimates. Identical proofs should also be used for higher order approximations below: we detail here every step. Denote $\chi_{\Omega}$ the characteristic function of the domain $\Omega, \delta_{\Gamma_{0}}$ the Dirac measure concentrated on $\Gamma_{0}$.

Proposition 1. There exist two positive constants $c_{1}$ and $c_{2}$, depending only of the mode $\hat{C}_{k}$ and the Sobolev's inequalities, such that:

$$
\left\|\hat{u}_{\epsilon, k}-\hat{u}_{0, k}\right\|_{H^{1}\left(\Omega_{\epsilon}\right)} \leq c_{1} \sqrt{\epsilon}, \quad\left\|\hat{u}_{\epsilon, k}-\hat{u}_{0, k}\right\|_{L^{2}\left(\Omega_{0}\right)} \leq c_{2} \epsilon
$$

Proof: The the first part of the proof is based on standard a priori estimates. The existence and uniqueness of $\hat{u}_{\epsilon, k}$ are well known and derive from the Lax-Milgram theorem. We focus on the error, namely we set 
$R_{0}^{\epsilon}:=\hat{u}_{\epsilon, k}-\hat{u}_{0, k}$. Since the extension $\hat{u}_{0, k}$ of $\hat{u}_{0, k}$ satisfies:

$$
\begin{cases}\mathcal{L}_{k} \hat{u}_{0, k}=\hat{C}_{k} \chi_{\Omega_{0}}+i k \mathcal{M}_{k} x_{2} \chi_{\Omega_{\epsilon} \backslash \Omega_{0}} & \text { in } \Omega_{\epsilon} \\ \hat{u}_{0, k}=0 & \text { on } \Gamma_{1} \\ \hat{u}_{0, k}=\mathcal{M}_{k} x_{2} & \text { on } \Gamma_{\epsilon}\end{cases}
$$

Then the zeroth order error solves:

$$
\begin{cases}\mathcal{L}_{k} R_{0}^{\epsilon}=\hat{C}_{k} \chi_{\Omega_{\epsilon} \backslash \Omega_{0}}-i k \mathcal{M}_{k} x_{2} \chi_{\Omega_{\epsilon} \backslash \Omega_{0}} & \text { in } \Omega_{\epsilon}, \\ R_{0}^{\epsilon}=0 & \text { on } \Gamma_{1}, \\ R_{0}^{\epsilon}=-\mathcal{M}_{k} x_{2} & \text { on } \Gamma_{\epsilon} .\end{cases}
$$

We remark that a part of the error comes from the source term localized in $\Omega_{\epsilon} \backslash \Omega_{0}$, and another part comes from the non homogeneous boundary term on $\Gamma_{\epsilon}$. We set the lift:

$$
s=-\mathcal{M}_{k} x_{2} \chi_{\Omega_{\epsilon} \backslash \Omega_{0}}, \quad \tilde{R}_{0}^{\epsilon}=R_{0}^{\epsilon}-s .
$$

Then:

$$
\begin{cases}\mathcal{L}_{k} \tilde{R}_{0}^{\epsilon}=\hat{C}_{k} \chi_{\Omega_{\epsilon} \backslash \Omega_{0}}+\mathcal{M}_{k} \delta_{\Gamma_{0}} & \text { in } \Omega_{\epsilon} \\ \tilde{R}_{0}^{\epsilon}=0 & \text { on } \Gamma_{1} \\ \tilde{R}_{0}^{\epsilon}=0 & \text { on } \Gamma_{\epsilon}\end{cases}
$$

where the derivatives are computed in the sense of distributions. Then, on one hand, using Poincaré inequality, we have:

$$
\begin{aligned}
\left|\int_{\Omega_{\epsilon}} \mathcal{L}_{k} \tilde{R}_{0}^{\epsilon} \overline{\tilde{R}_{0}^{\epsilon}} d x\right|^{2} & =\left|i k\left\|\tilde{R}_{0}^{\epsilon}\right\|_{L^{2}\left(\Omega_{\epsilon}\right)}^{2}+\left\|\nabla \tilde{R}_{0}^{\epsilon}\right\|_{L^{2}\left(\Omega_{\epsilon}\right)}^{2}\right|^{2} \\
& =k^{2}\left\|\tilde{R}_{0}^{\epsilon}\right\|_{L^{2}\left(\Omega_{\epsilon}\right)}^{4}+\left\|\nabla \tilde{R}_{0}^{\epsilon}\right\|_{L^{2}\left(\Omega_{\epsilon}\right)}^{4} \\
& \geq c\left\|\tilde{R}_{0}^{\epsilon}\right\|_{H^{1}\left(\Omega_{\epsilon}\right)}^{4} .
\end{aligned}
$$

And on the other hand, for any test function $\phi \in H_{0}^{1}\left(\Omega_{\epsilon}\right)$ :

$$
\int_{\Omega_{\epsilon}} \mathcal{L}_{k} \tilde{R}_{0}^{\epsilon} \phi d x=\hat{C}_{k} \int_{\Omega_{\epsilon} \backslash \Omega_{0}} \phi d x+\mathcal{M}_{k} \int_{\Gamma_{0}} \phi d x
$$

Then, using Cauchy-Schwarz and Poincaré like inequalities, we obtain the upper bound:

$$
\left|i k\left\|\tilde{R}_{0}^{\epsilon}\right\|_{L^{2}\left(\Omega_{\epsilon}\right)}^{2}+\left\|\nabla \tilde{R}_{0}^{\epsilon}\right\|_{L^{2}\left(\Omega_{\epsilon}\right)}^{2}\right| \leq c\left(\left|\hat{C}_{k}\right| \epsilon+\left|\mathcal{M}_{k}\right| \sqrt{\epsilon}\right)\left\|\tilde{R}_{0}^{\epsilon}\right\|_{H^{1}\left(\Omega_{\epsilon}\right)},
$$

where $c$ is a non negative constant depending on the Poincaré inequality. And $\left|\mathcal{M}_{k}\right|$ is controlled as follows:

$$
\left|\mathcal{M}_{k}\right|=\frac{\left|\hat{C}_{k} r\left(2-e^{r}-e^{-r}\right)\right|}{\left|i k\left(e^{r}-e^{-r}\right)\right|} \leq \frac{\left|\hat{C}_{k}\right|}{\sqrt{k}}\left(\frac{2}{\left|e^{r}-e^{-r}\right|}+1\right) \leq 2\left|\hat{C}_{k}\right| .
$$

Finally, combining (10)-(12), we get the $H^{1}$-error estimate.

For the $L^{2}$ error, we use the concept of a very weak solution. Namely, one solves the dual problem: for a given $\phi \in L^{2}\left(\Omega_{0}\right), \phi$ being $x_{1}$ periodic on $\Gamma_{\text {in }} \cup \Gamma_{\text {out }}$, find $\hat{v} \in H^{2}\left(\Omega_{0}\right)$ such that

$$
\begin{cases}\overline{\mathcal{L}}_{k} \hat{v}=\phi & \text { in } \Omega_{0} \\ \hat{v}=0 & \text { on } \Gamma_{1} \cup \Gamma_{0} \\ \hat{v} \text { is } x_{1} \text {-periodic } & \text { on } \Gamma_{\text {in }} \cup \Gamma_{\text {out }}\end{cases}
$$


Then, considering the $L^{2}\left(\Omega_{0}\right)$ scalar product $(.,$.$) , and using the Green formula:$

$$
\begin{aligned}
\left(R_{0}^{\epsilon}, \phi\right)=\int_{\Omega_{0}} R_{0}^{\epsilon} \overline{\mathcal{L}}_{k} \hat{v}=-i k \int_{\Omega_{0}} R_{0}^{\epsilon} \hat{v}+\int_{\Omega_{0}} \nabla R_{0}^{\epsilon} \nabla \hat{v}-\int_{\partial \Omega_{0}} R_{0}^{\epsilon} \frac{\partial \hat{v}}{\partial n} & =\left\langle\hat{v}, \frac{\partial R_{0}^{\epsilon}}{\partial n}\right\rangle_{\Gamma_{\text {in }} \cup \Gamma_{\text {out }}}-\left(R_{0}^{\epsilon}, \frac{\partial \hat{v}}{\partial n}\right)_{\Gamma_{0} \cup \Gamma_{1}},
\end{aligned}
$$

where the brackets refer to the dual product in $\left(H^{-1}, H^{1}\right)\left(\partial \Omega_{0}\right)$ and the rest of the products are in $L^{2}$ either on $\Gamma_{0}$ or on $\Omega_{0}$. Then one computes:

$$
\left|\left(R_{0}^{\epsilon}, \phi\right)\right| \leq\left\|R_{0}^{\epsilon}\right\|_{L^{2}\left(\Gamma_{0}\right)}\left\|\frac{\partial \hat{v}}{\partial n}\right\|_{L^{2}\left(\Gamma_{0}\right)} \leq \sqrt{\epsilon}\left\|\nabla R_{0}^{\epsilon}\right\|_{L^{2}\left(\Omega_{\epsilon} \backslash \Omega_{0}\right)}\|\phi\|_{L^{2}\left(\Omega_{0}\right)} \leq \sqrt{\epsilon}\left\|\nabla R_{0}^{\epsilon}\right\|_{L^{2}\left(\Omega_{\epsilon}\right)}\|\phi\|_{L^{2}\left(\Omega_{0}\right)} \leq \epsilon^{\frac{3}{2}}\|\phi\|_{L^{2}\left(\Omega_{0}\right)} .
$$

And this ends the proof of the proposition, by taking the sup over all $\phi \in L^{2}\left(\Omega_{0}\right)$. But between the first and the second estimate above, we assumed that the solutions of the regular problem (13) satisfy a kind of Rellich estimates (see [12], chap. 5) :

$$
\left\|\frac{\partial \hat{v}}{\partial n}\right\|_{L^{2}\left(\Gamma_{0}\right)} \leq c\|\phi\|_{L^{2}\left(\Omega_{0}\right)}
$$

In order to prove this, we decompose $\phi$ on the Hilbert basis $\left(e^{2 \pi i n x_{1}} e^{2 \pi i m x_{2}}\right)_{n, m}$ of $L^{2}\left(\Omega_{0}\right)$. Separating the variables, define $\phi_{n}\left(x_{2}\right)$ the coordinates of $\phi$ in the $\left(e^{2 \pi i n x_{1}}\right)_{n}$ Hilbert basis of $L^{2}(0,1)$, and $a_{n, m}$ its coordinates in the basis $\left(e^{2 \pi i n x_{1}} e^{2 \pi i m x_{2}}\right)_{n, m}$. Then $\phi$ can be written under the form:

$$
\phi=\sum_{n \in \mathbb{Z}} \phi_{n}\left(x_{2}\right) e^{2 \pi i n x_{1}}=\sum_{n, m \in \mathbb{Z}} a_{n, m} e^{2 \pi i n x_{1}} e^{2 \pi i n x_{2}}, \quad \text { and }\|\phi\|_{L^{2}\left(\Omega_{0}\right)}^{2}=\sum_{n, m \in \mathbb{Z}}\left|a_{n, m}\right|^{2} .
$$

In the same way, one can decompose $\hat{v}$ on the basis: $\hat{v}=\sum_{n \in \mathbb{Z}} \hat{v}_{n}\left(x_{2}\right) e^{2 \pi i n x_{1}}$. Then the first equation of system (13) can be rewritten under the form of an infinite system of ordinary differential equations:

$$
\forall l \in \mathbb{Z}, \quad\left(i k+4 \pi^{2} l^{2}\right) \hat{v}_{l}-\hat{v}_{l}^{\prime \prime}=\phi_{l} .
$$

And the solution, for a fixed $l$, is given by:

$$
\hat{v}_{l}=A e^{b x_{2}}+B e^{-b x_{2}}+\hat{v}_{p, l},
$$

where $\hat{v}_{p, l}$ stands for the particular solution and reads

$$
\hat{v}_{p, l}:=\sum_{m \in \mathbb{Z}} \frac{-a_{l, m}}{4 \pi^{2} m^{2}+b^{2}} e^{2 \pi i m x_{2}}, \quad A-B=\tanh (b) \hat{v}_{p, l}(0)-\frac{\hat{v}_{p, l}(1)}{\sinh (b)}, \quad b^{2}=4 \pi^{2} l^{2}+i k,
$$

Then, since

it remains to estimate:

$$
\left\|\frac{\partial \hat{v}}{\partial n}\right\|_{L^{2}\left(\Gamma_{0}\right)}^{2}=\sum_{l \in \mathbb{Z}}\left|\hat{v}_{l}^{\prime}(0)\right|^{2},
$$

$$
\left|\hat{v}_{l}^{\prime}(0)\right|^{2}=\left|b(A-B)+\hat{v}_{p, l}^{\prime}(0)\right|^{2} \leq 2\left(|b(A-B)|^{2}+\left|\hat{v}_{p, l}^{\prime}(0)\right|^{2}\right) .
$$

The intermediate variable $b$ solves in $\mathbb{C}$ the equation $b^{2}=4 \pi^{2} l^{2}+i k$ which implies that

$$
b:=b_{r}+i b_{i}, \quad b_{r}:= \pm \sqrt{2 \pi^{2} l^{2}+\sqrt{4 \pi^{4} l^{4}+k^{2}}}, \quad b_{i}:= \pm \sqrt{-2 \pi^{2} l^{2}+\sqrt{4 \pi^{4} l^{4}+k^{2}}},
$$


so that

$$
|b|^{2}=b_{r}^{2}+b_{i}^{2}=2 \sqrt{4 \pi^{4} l^{4}+k^{2}} \geq 2, \quad \forall l \in \mathbb{Z}, \quad \forall k \in \mathbb{Z}^{*}
$$

We return to the rhs of (16), the first term of the rhs can be split in two parts:

$$
|b|^{2}\left|\hat{v}_{p, l}(0)\right|^{2}|\tanh (b)|^{2} \leq\left|\sum_{m} \frac{a_{m, l}}{4 \pi^{2} m^{2}+b^{2}}\right|^{2}|b|^{2}|\tanh (b)|^{2} \leq 2\left(\sum_{m}\left|a_{m, l}\right|^{2}\right)\left(\sum_{m} \frac{|b|^{2}}{4 \pi^{4} m^{4}+|b|^{4}}\right)|\tanh (b)|^{2}
$$

for sake of conciseness we set:

then it is equivalent to write

$$
I:=\left(\sum_{m \in \mathbb{Z}} \frac{|b|^{2}}{4 \pi^{4} m^{4}+|b|^{4}}\right)
$$

$$
I=\frac{1}{|b|^{2}}+2 \sum_{m \geq 1} \frac{|b|^{2}}{4 \pi^{4} m^{4}+|b|^{4}}=: I_{1}+I_{2}
$$

If $x$ is a positive real, we set $m:=E[x]$ where $E[\cdot]$ is the integer part of its argument, one then has

$$
I_{2} \leq \int_{1}^{\infty} \frac{|b|^{2}}{4 \pi^{4}(x-1)^{4}+|b|^{4}} d x=\int_{0}^{\infty} \frac{|b|^{2}}{4 \pi^{4} x^{4}+|b|^{4}} d x \leq \int_{0}^{1}+\int_{1}^{\infty} \frac{|b|^{2}}{4 \pi^{4} x^{4}+|b|^{4}} d x \leq c\left(\frac{1}{|b|^{2}}+\frac{1}{|b|}\right)
$$

so that finally $I \leq c$, the constant $c$ being independent on either $l$ or $k$. Because $b_{r} \neq 0$ one has that

$$
|\tanh (b)|=\frac{e^{2 b_{r}}+e^{-2 b_{r}}+2 \cos \left(b_{i}\right)}{e^{2 b_{r}}+e^{-2 b_{r}}-2 \cos \left(b_{i}\right)} \leq \frac{4+\sum_{q=1}^{\infty} \frac{\left(2 b_{r}\right)^{2 q}}{(2 q) !}}{\sum_{q=1}^{\infty} \frac{\left(2 b_{r}\right)^{2 q}}{(2 q) !}} \leq c
$$

where c does not depend on $k$ nor on $l$. In a similar way one gets again using Cauchy-Schwartz

$$
\frac{\left|\hat{v}_{p, l}(1)\right|^{2}|b|^{2}}{|\sinh (b)|^{2}} \leq \sum_{m}\left|a_{m, l}\right|^{2}\left(\sum_{m} \frac{|b|^{2}}{4 \pi^{4} m^{4}+|b|^{4}}\right) \frac{1}{|\sinh (b)|^{2}} \leq c\left\|\phi_{l}\right\|_{L^{2}(0,1)}^{2}
$$

where again $c$ is a generic constant independent on $k, l$. The estimates of $\left|\hat{v}_{p, l}^{\prime}(0)\right|$ follow the same lines.

\subsubsection{First order correction}

We have already seen that the zeroth order approximation contains two distinct sources of errors: a part is due to the order of the extension $\hat{u}_{0, k}$ in $\Omega_{\epsilon} \backslash \Omega_{0}$ and another part comes from the non homogeneous rest on $\Gamma_{\epsilon}$. In order to correct the non zero value of $\hat{u}_{0, k}$ on the rough boundary $\Gamma_{\epsilon}$, we introduce the corrector $\beta$, defined on the microscopic cell $Z^{+} \cup \Gamma \cup P$

$$
\begin{cases}\Delta \beta=0 & \text { in } Z^{+} \cup P \\ \beta=-y_{2} & \text { on } P_{0} \\ \beta \text { is } y_{1} \text {-periodic. } & \end{cases}
$$

We define the microscopic average along the fictitious interface $\Gamma$ :

$$
\bar{\beta}=\frac{1}{2 \pi} \int_{0}^{2 \pi} \beta\left(y_{1}, 0\right) d y_{1} .
$$

The existence and uniqueness of $\beta$, and its properties, as the exponential convergence towards $\bar{\beta}$ when $y_{2}$ tends to infinity, are described in [2] and references therein. Because $\beta$ tends to $\bar{\beta}$ when $y_{2}$ goes to infinity, we subtract 
this constant in the final asymptotic ansatz. As the constant should be relevant only far from the roughness we correct the ansatz by adding $\hat{u}_{1, k}$ a "counter-flow" approximation solving:

$$
\begin{cases}\mathcal{L}_{k} \hat{u}_{1, k}=0 & \text { in } \Omega_{0} \\ \hat{u}_{1, k}=0 & \text { on } \Gamma_{1} \\ \hat{u}_{1, k}=\bar{\beta} \mathcal{M}_{k} & \text { on } \Gamma_{0} \\ \hat{u}_{1, k} \text { is } x_{1} \text {-periodic } & \text { on } \Gamma_{\text {in }} \cup \Gamma_{\text {out }}\end{cases}
$$

The solution is explicit:

$$
\hat{u}_{1, k}=\frac{-\bar{\beta} \mathcal{M}_{k}}{e^{r}-e^{-r}}\left(e^{-r} e^{r x_{2}}-e^{r} e^{-r x_{2}}\right)=\bar{\beta} \mathcal{M}_{k} \frac{\sinh \left(r\left(1+x_{2}\right)\right)}{\sinh (r)}
$$

Now we are in the position to define the full boundary layer approximation :

$$
\hat{\mathcal{U}}_{\epsilon, k}:=\hat{u}_{0, k}+\epsilon \mathcal{M}_{k}\left(\beta\left(\frac{x}{\epsilon}\right)-\bar{\beta}\right)+\epsilon \hat{u}_{1, k}
$$

\subsubsection{First order estimates}

The gain obtained when introducing the microscopic corrector is of order $\sqrt{\epsilon}$. Indeed, the following error estimates hold.

Proposition 2. There exist two positive constants $c_{3}$ and $c_{4}$, depending only on the mode $\hat{C}_{k}$ and not on the frequency $k$, such that:

$$
\left\|\hat{u}_{\epsilon, k}-\hat{\mathcal{U}}_{\epsilon, k}\right\|_{H^{1}\left(\Omega_{\epsilon}\right)} \leq c_{3} \epsilon, \quad\left\|\hat{u}_{\epsilon, k}-\hat{\mathcal{U}}_{\epsilon, k}\right\|_{L^{2}\left(\Omega_{0}\right)} \leq c_{4} \epsilon^{3 / 2}
$$

Proof: Denote $R_{\epsilon}:=\hat{u}_{\epsilon, k}-\hat{\mathcal{U}}_{\epsilon, k}$ the error to estimate. It is solution of the problem:

$$
\begin{cases}\mathcal{L}_{k} R_{\epsilon}=\hat{C}_{k} \chi_{\Omega_{\epsilon} \backslash \Omega_{0}}-i k \mathcal{M}_{k} x_{2} \chi_{\Omega_{\epsilon} \backslash \Omega_{0}}-i k \mathcal{M}_{k} \epsilon\left(\beta\left(\frac{x}{\epsilon}\right)-\bar{\beta}+\bar{\beta} \chi_{\Omega_{\epsilon} \backslash \Omega_{0}}\right)-\epsilon \mathcal{M}_{k} \bar{\beta} \delta_{\Gamma_{0}}, & \text { in } \Omega_{\epsilon} \\ R_{\epsilon}=-\epsilon \mathcal{M}_{k}\left(\beta\left(\frac{x}{\epsilon}, \frac{1}{\epsilon}\right)-\bar{\beta}\right) & \text { on } \Gamma_{1} \\ R_{\epsilon}=0 & \text { on } \Gamma_{\epsilon} \\ R_{\epsilon} \text { is } x_{1} \text {-periodic on } \Gamma_{\text {in }} \cup \Gamma_{\text {out }} & \end{cases}
$$

The existence and uniqueness of $R_{\epsilon}$ are standard. We focus again on the a priori estimates: test the system above by $\bar{R}_{\epsilon}$ and estimate the lhs from below as in (10), then estimate from above the rhs. The last step includes new terms wrt the zeroth order approximation :

$$
\left\{\begin{array}{l}
A_{1}=\hat{C}_{k} \int_{\Omega_{\epsilon} \backslash \Omega_{0}} \bar{R}_{\epsilon} d x, \quad A_{2}=-i k \mathcal{M}_{k} \int_{\Omega_{\epsilon} \backslash \Omega_{0}} x_{2} \bar{R}_{\epsilon} d x, \quad A_{3}=-i k \mathcal{M}_{k} \epsilon \int_{\Omega_{\epsilon}}\left(\beta\left(\frac{x}{\epsilon}\right)-\bar{\beta}\right) \bar{R}_{\epsilon} d x \\
A_{4}=-i k \mathcal{M}_{k} \epsilon \bar{\beta} \int_{\Omega_{\epsilon} \backslash \Omega_{0}} \bar{R}_{\epsilon} d x, \quad A_{5}=-\epsilon \overline{\mathcal{M}} \int_{\Gamma_{0}} \bar{R}_{\epsilon} d x_{1}
\end{array}\right.
$$

Then, estimating these terms, one gets

$$
\left|\sum_{j=1}^{5} A_{j}\right| \leq \epsilon^{\frac{3}{2}} c\left\|\nabla R_{\epsilon}\right\|_{L^{2}\left(\Omega_{\epsilon}\right)}
$$

which ends the proof for the a priori estimates. Again very weak estimates give:

$$
\left\|R_{\epsilon}\right\|_{L^{2}\left(\Omega_{0}\right)} \leq\left\|R_{\epsilon}\right\|_{L^{2}\left(\Gamma_{1} \cup \Gamma_{0}\right)}+\epsilon\left|k \mathcal{M}_{k}\right|\left\|\beta\left(\frac{\dot{-}}{\epsilon}\right)-\bar{\beta}\right\|_{L^{2}\left(\Omega_{0}\right)} \leq c\left(e^{-\frac{1}{\epsilon}}+\sqrt{\epsilon}\left\|\nabla R_{\epsilon}\right\|_{L^{2}\left(\Omega_{\epsilon} \backslash \Omega_{0}\right)}+\epsilon^{\frac{3}{2}}\right) \leq c \epsilon^{\frac{3}{2}}
$$




\subsubsection{Derivation of Wall-laws}

Averaging the ansatz. We aim to derive a system of equations defined on the smooth domain $\Omega_{0}$, for which the effect of the roughness is included as a macroscopic boundary condition on $\Gamma_{0}$. First, averaging wrt the fast variable in the horizontal direction, we get:

$$
\overline{\hat{\mathcal{U}}_{\epsilon, k}}=\hat{u}_{0, k}+\epsilon \hat{u}_{1, k}:=\bar{u}_{\epsilon, k} .
$$

Though, the averaging process cancels the oscillations, the averaged ansatz still contains a first order macroscopic correction $\hat{u}_{1, k}$ accounting for averaged first order effects. This new averaged quantity solves a problem in the smooth limiting domain $\Omega_{0}$ :

$$
\begin{cases}\mathcal{L}_{k} \bar{u}_{\epsilon, k}=\hat{C}_{k} & \text { in } \Omega_{0}, \\ \bar{u}_{\epsilon, k}=0 & \text { on } \Gamma_{1}, \\ \bar{u}_{\epsilon, k}=\epsilon \mathcal{M}_{k} \bar{\beta} & \text { on } \Gamma_{0}, \\ \bar{u}_{\epsilon, k} \text { is } x_{1} \text {-periodic } & \text { on } \Gamma_{\text {in }} \cup \Gamma_{\text {out }} .\end{cases}
$$

We compute the $L^{2}$-error estimate between the exact solution $\hat{u}_{\epsilon, k}$ of problem (3) and the averaged first order approximation $\bar{u}_{\epsilon, k}$.

Proposition 3. There exists one positive constant $c_{5}$, depending only of the mode $\hat{C}_{k}$ such that:

$$
\left\|\hat{u}_{\epsilon, k}-\bar{u}_{\epsilon, k}\right\|_{L^{2}\left(\Omega_{0}\right)} \leq c_{5} \epsilon^{3 / 2}
$$

Proof: We write a triangular inequality:

$$
\left\|\hat{u}_{\epsilon, k}-\bar{u}_{\epsilon, k}\right\|_{L^{2}\left(\Omega_{0}\right)} \leq\left\|\hat{u}_{\epsilon, k}-\hat{\mathcal{U}}_{\epsilon, k}\right\|_{L^{2}\left(\Omega_{0}\right)}+\left\|\hat{\mathcal{U}}_{\epsilon, k}-\bar{u}_{\epsilon, k}\right\|_{L^{2}\left(\Omega_{0}\right)} .
$$

The second term in the rhs is explicit :

$$
\hat{\mathcal{U}}_{\epsilon, k}-\bar{u}_{\epsilon, k}=\epsilon \mathcal{M}_{k}\left(\beta\left(\frac{x}{\epsilon}\right)-\bar{\beta}\right) .
$$

One thus estimates this quantity directly in the $L^{2}\left(\Omega_{0}\right)$ norm. Thanks to the multi-scale structure of this corrector one gets by a simple change of variable and thanks to the specific boundary layer properties of $\beta$ that

$$
\left\|\beta\left(\frac{\cdot}{\epsilon}\right)-\bar{\beta}\right\|_{L^{2}\left(\Omega_{0}\right)} \leq \sqrt{\epsilon}\|\beta-\bar{\beta}\|_{L^{2}(Z+\cup \Gamma \cup P)}
$$

which ends the proof.

\subsubsection{Implicit wall-law}

In order to derive an implicit wall-law, we rewrite the boundary condition satisfied by $\bar{u}_{\epsilon, k}$ on $\Gamma_{0}$ :

$$
\bar{u}_{\epsilon, k}=\epsilon \mathcal{M}_{k} \bar{\beta}=\epsilon \bar{\beta} \frac{\partial}{\partial x_{2}}\left(\hat{u}_{0, k}+\epsilon \hat{u}_{1, k}-\epsilon \hat{u}_{1, k}\right)=\epsilon \bar{\beta} \frac{\partial \bar{u}_{\epsilon, k}}{\partial x_{2}}-\epsilon^{2} \bar{\beta} \frac{\partial \hat{u}_{1, k}}{\partial x_{2}} \quad \text { on } \Gamma_{0} .
$$

Hence, since the term $\partial \hat{u}_{1, k} / \partial x_{2}$ can be bounded independently from the frequency $k$, we derive a first order implicit wall-law. Indeed,

So, when $k \neq 1$ :

$$
\frac{\partial \hat{u}_{1, k}}{\partial x_{2}}\left(x_{2}=0\right)=-\hat{C}_{k}\left(\frac{e^{r}+e^{-r}}{e^{r}-e^{-r}}\right)^{2} .
$$

$$
\left|\frac{\partial \hat{u}_{1, k}}{\partial x_{2}}\left(x_{2}=0\right)\right| \leq\left|\hat{C}_{k}\right|\left(\frac{1}{1-e^{-\sqrt{2}}}\right)^{2} .
$$


We set the following approximate problem, posed in the smooth domain $\Omega_{0}$ :

$$
\begin{cases}\mathcal{L}_{k} \hat{V}_{\epsilon, k}=\hat{C}_{k} & \text { in } \Omega_{0}, \\ \hat{V}_{\epsilon, k}=0 & \text { on } \Gamma_{1}, \\ \hat{V}_{\epsilon, k}=\epsilon \bar{\beta} \frac{\partial \hat{V}_{\epsilon, k}}{\partial x_{2}} & \text { on } \Gamma_{0}, \\ \hat{V}_{\epsilon, k} \text { is } x_{1} \text {-periodic } & \text { on } \Gamma_{\text {in }} \cup \Gamma_{\text {out }} .\end{cases}
$$

It remains to show that this first order implicit wall-law has a solution and is an approximation in the smooth domain $\Omega_{0}$ of the rough problem (3). The existence of solution in $H_{\Gamma_{1}}^{1}\left(\Omega_{0}\right)\left(H^{1}\right.$-functions vanishing on $\left.\Gamma_{1}\right)$ for problem (28) is not discussed here (see for example [2]), but the error estimate are given in the following theorem.

Theorem 1.1. There exists two positive constants $c_{6}$ and $c_{7}$, depending only of the mode $\hat{C}_{k}$ and not on the frequency $k$ such that:

$$
\left\|\hat{u}_{\epsilon, k}-\hat{V}_{\epsilon, k}\right\|_{L^{2}\left(\Omega_{0}\right)} \leq c_{6} \epsilon^{3 / 2} \text { and } \quad\left\|\hat{u}_{\epsilon, k}-\hat{V}_{\epsilon, k}\right\|_{H^{1}\left(\Omega_{0}\right)} \leq c_{7} \sqrt{\epsilon}
$$

Proof: We split the error into two parts:

$$
\left\|\hat{u}_{\epsilon, k}-\hat{V}_{\epsilon, k}\right\|_{L^{2}\left(\Omega_{0}\right)} \leq\left\|\hat{u}_{\epsilon, k}-\bar{u}_{\epsilon, k}\right\|_{L^{2}\left(\Omega_{0}\right)}+\left\|\bar{u}_{\epsilon, k}-\hat{V}_{\epsilon, k}\right\|_{L^{2}\left(\Omega_{0}\right)}
$$

The first term is controlled thanks to Proposition 3. For the second one, let us define $\Theta:=\bar{u}_{\epsilon, k}-\hat{V}_{\epsilon, k}$ and consider the boundary value problem it satisfies:

$$
\begin{cases}\mathcal{L}_{k} \Theta=0 & \text { in } \Omega_{0}, \\ \Theta=0 & \text { on } \Gamma_{1} \\ \Theta=\epsilon \bar{\beta}\left(\frac{\partial \hat{u}_{0, k}}{\partial x_{2}}-\frac{\partial \hat{V}_{\epsilon, k}}{\partial x_{2}}\right) & \text { on } \Gamma_{0} \\ \Theta \text { is } x_{1} \text {-periodic } & \text { on } \Gamma_{\text {in }} \cup \Gamma_{\text {out }} .\end{cases}
$$

We re-express the boundary condition on $\Gamma_{0}$ introducing a Robin like condition, namely:

$$
\Theta-\epsilon \bar{\beta} \frac{\partial \Theta}{\partial x_{2}}=\epsilon \bar{\beta}\left(\frac{\partial \hat{u}_{0, k}}{\partial x_{2}}-\frac{\partial \bar{u}_{\epsilon, k}}{\partial x_{2}}\right)=-\epsilon^{2} \bar{\beta} \frac{\partial \hat{u}_{1, k}}{\partial x_{2}} \text { on } \Gamma_{0}
$$

where the rhs is now explicitly known. One sets

$$
a_{k}(\theta, v)=(\nabla \theta, \nabla v)_{\Omega_{0}}+i k(\theta, v)_{\Omega_{0}}+\left(\frac{\theta}{\epsilon \bar{\beta}}, v\right)
$$

and it is easy to show that this bi-linear form is bi-continuous and coercive. The variational problem becomes now

which gives directly by a priori estimates that

$$
a_{k}(\theta, v)=-\epsilon^{2}\left(\frac{\partial \hat{u}_{0, k}}{\partial x_{2}}, v\right)_{\Gamma_{0}}, \quad \forall v \in H_{\Gamma_{1}}^{1}\left(\Omega_{0}\right),
$$

$$
\|\nabla \theta\|_{L^{2}\left(\Omega_{0}\right)} \leq c \epsilon^{2}, \quad\|\theta\|_{L^{2}\left(\Gamma_{0}\right)} \leq c \epsilon^{3}
$$

One then uses the very weak estimates in order to estimate $\theta$ in the $L^{2}\left(\Omega_{0}\right)$ norm and concludes thanks to the last trace estimate. For the a priori part we simply decompose the error using every result established above 
to get:

$$
\begin{aligned}
\left\|\hat{u}_{\epsilon, k}-\hat{V}_{\epsilon, k}\right\|_{H^{1}\left(\Omega_{0}\right)} & \leq\left\|\hat{u}_{\epsilon, k}-\hat{\mathcal{U}}_{\epsilon, k}\right\|_{H^{1}\left(\Omega_{0}\right)}+\left\|\hat{\mathcal{U}}_{\epsilon, k}-\bar{u}_{\epsilon, k}\right\|_{H^{1}\left(\Omega_{0}\right)}+\left\|\bar{u}_{\epsilon, k}-\hat{V}_{\epsilon, k}\right\|_{H^{1}\left(\Omega_{0}\right)} \\
& \leq\left\|\hat{u}_{\epsilon, k}-\hat{\mathcal{U}}_{\epsilon, k}\right\|_{H^{1}\left(\Omega_{\epsilon}\right)}+c \sqrt{\epsilon}\left\|\nabla_{y} \beta\right\|_{L^{2}\left(Z^{+} \cup \Gamma \cup P\right)}+\epsilon^{2} \leq c\left(\epsilon+\sqrt{\epsilon}+\epsilon^{2}\right) \leq c^{\prime} \sqrt{\epsilon} .
\end{aligned}
$$

\subsection{Numerical results}

\subsubsection{Discretization}

In this part we aim to prove numerically that wall-laws perform better approximation than the zeroth order guess. For this sake we define an explicit shape of the roughness setting $f$ in (1) to be :

$$
f\left(y_{1}\right):=-\frac{\left(1+\cos \left(y_{1}\right)\right)}{2}-\delta,
$$

with $\delta$ being a positive constant equal to $5 \cdot 10^{-2}$. The periodicity of the bottom shape and of the boundary conditions on $\Gamma_{\text {in }} \cup \Gamma_{\text {out }}$ allows to discretize only a single rough period, i.e. we set

$$
\left.\Omega_{\#, \epsilon,-}:=\left\{x_{1} \in\right] 0,2 \pi \epsilon\left[\text { and } x_{2} \in\right] \epsilon f\left(x_{1} / \epsilon\right), 0[\}, \quad \Omega_{\#, \epsilon,+}:=\right] 0,2 \pi \epsilon[\times] 0,1\left[, \quad \Omega_{\#, \epsilon}:=\Omega_{\#, \epsilon,+} \cup \Omega_{\#, \epsilon,-},\right.
$$
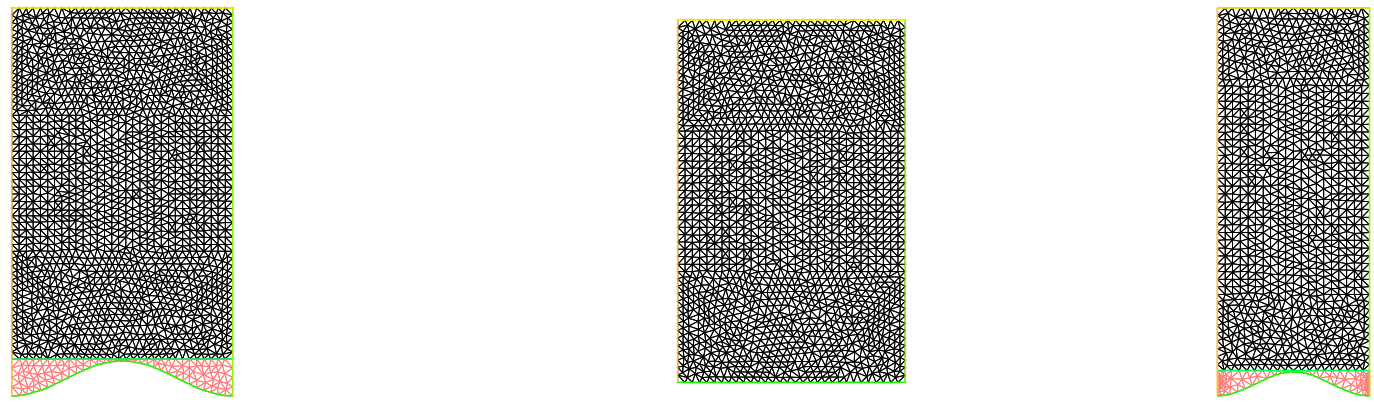

Figure 3. Meshes $\Omega_{\#, \epsilon}, \Omega_{\#, \epsilon,+}$, when $\epsilon=0.1$ and $Z^{+} \cup \Gamma \cup P \cap\left\{y_{2} \leq L=10\right\}$ (coarse grids, see below for actual mesh sizes)

The mesh is periodic, i.e. the vertices on $\Gamma_{\text {in }}$ are associated to elements containing edges on $\Gamma_{\text {out }}$ (see p. 142 of the freefem ++ documentation for further information on this facility). For a given $\epsilon$, the meshes of $\Omega_{\#, \epsilon}$ and $\Omega_{\#, 0}$ are conforming on the upper part $\left\{x_{2} \geq 0\right\}$. We take several values of $\epsilon$, namely we set $i \in\{1, \ldots, 10\}$ and $\epsilon=q^{i}$, where $q:=0.85$. In order to avoid discretization errors we set $n^{\epsilon}:=90 / \epsilon^{\alpha}, \alpha=0.2$ nodes on the horizontal fictitious boundary, and linearly proportional numbers of nodes on the other boundaries. This gives a mesh size $h$ (maximal diameter of a triangulation, see p.88 [5]) depicted in fig. 4 (right) as a function of $\epsilon$. Thus there exists a constant $c$ independent of $\epsilon$ such that $h \leq c \epsilon$. We fix a frequency $k=10$ for which $\hat{C}_{k} \equiv 10$, we compute numerical approximations of

- $\hat{u}_{\epsilon, k, h}$ solving the discretized problem (3)

- $\hat{u}_{0, k, h}$, the zeroth order Poiseuille-like approximation solving system (4)

- $\hat{V}_{\epsilon, k, h}$, the implicit discrete wall-law. 
In order to compute the cell problem and $\bar{\beta}$, the constant at infinity related to the specific roughness $f$, we discretize a cell problem defined on a truncated domain : find $\beta_{L}$ solving

$$
\left\{\begin{array}{lr}
-\Delta \beta_{L}=0 & \text { in } Z^{+} \cup \Gamma \cup P \cap\left\{y_{2}<L\right\} \\
\partial_{\mathbf{n}} \beta=0 & \text { on }\left\{y_{2}=L\right\} \\
\beta=-y_{2} & \text { on } P_{0}
\end{array}\right.
$$

It is shown in [8] that the solution $\beta_{L}$ converges exponentially fast, when $L \rightarrow \infty$, towards the solution of (17). So solving the problem above provides a good approximation of $\bar{\beta}_{L}$. And we use this numerical value in the boundary condition on $\Gamma_{0}$ in (28) in order to compute $\hat{V}_{\epsilon, k, h}$. The code is written in freefem++ language [5]: it is very well suited for solving complex valued variational problems with finite elements. Our code is available through Internet ${ }^{1}$.

\subsubsection{Results}

We compute numerical equivalent norms for a priori and very weak estimates. We plot this results in the $\log -\log$ scale for various sizes $\epsilon$ (in abscissa) in fig. 4. We recover better orders of convergence than expected:
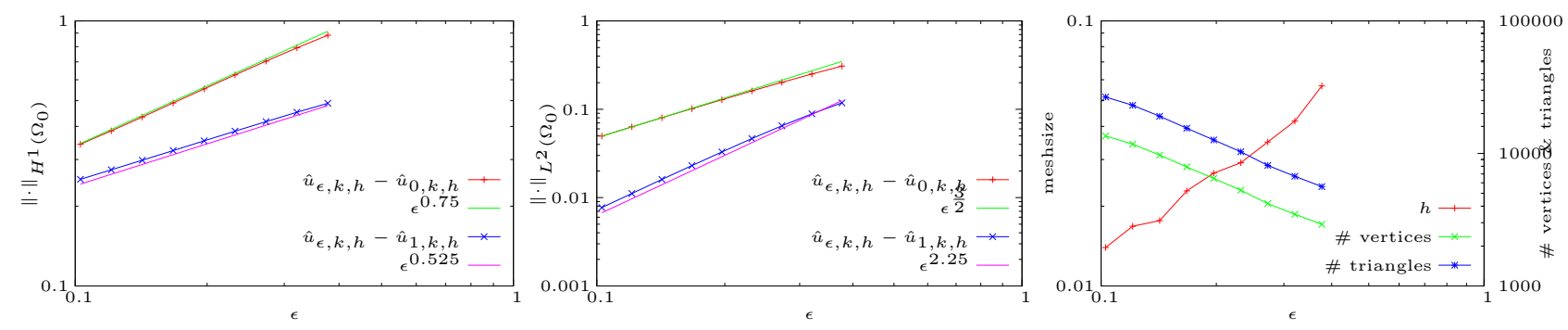

FigurE 4. Numerical error estimates in $H^{1}\left(\Omega_{0}\right)$ (left) and $L^{2}\left(\Omega_{0}\right)$ (middle) norms, and mesh parameters (right)

the very weak estimates provide $\epsilon^{\frac{3}{2}}$ convergence for the Poiseuille profile while they give $\epsilon^{\frac{9}{4}}$ for the wall-law. The $H^{1}\left(\Omega_{0}\right)$ norm $\left(\sim \epsilon^{\frac{3}{4}}\right)$ is better than expected for the Poiseuille profile while surprisingly the error is worse for the wall-law wrt this norm. This is due to the $\sqrt{\epsilon}$ Dirichlet norm of gradient of boundary layers as already shown in Theorem 1.1. This numerical test shows that for this geometry case this latter estimate is almost optimal.

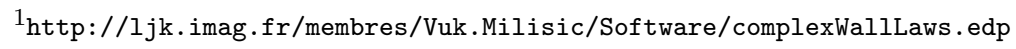




\section{Numerical INVESTigation}

\subsection{Saccular side aneurysm}

The objective of this numerical experiment is to determine actual computational limits, when running threedimensional blood flow simulations of the non-homogenized stented vessels. Extremely small wire cross-section, $\varnothing=0.1 \mathrm{~mm}$, complex, almost random, spacing between braided wires could not be properly modeled in actual computational reality. Even when such models have been developed, industrial computer aided design (CAD) programs, mesh generators and finite element analysis tools are not well optimized for processing complex freeform geometries. However, it is important to analyze modeling and discretization limits, spatial resolution, memory needs and computation time required to guarantee an accurate and reliable hemodynamic simulation of stented vessels. The authors hope that the sequence of direct simulations brings an additional design insight, and could be used as a reference solution for the further three-dimensional homogenization research.

Parametric, three-dimensional model of a blood vessel with a side saccular aneurysm was built using commercial software, CATIA V5. The aneurysm has an ellipsoidal extended shape of $22 \mathrm{~mm}$ over $17 \mathrm{~mm}$ across its largest and smallest diameters, respectively. It is attached to the parent vessel of $60 \mathrm{~mm}$ long within a constant diameter of $10 \mathrm{~mm}$, fig. 5, (left). These values are representative of the femoral artery. A braided tabular assembly of thin metallic wires is lodged against the lumen of the vessel to serve as a porous barrier disrupting blood flow into the aneurysm, fig. 5, (right). A finite element model of a two-layer stent, braided of 32 wires has been built, fig. 5, (bottom). Similar to commercial stents, but not being an exact replica, it has a $10 \mathrm{~mm}$ diameter, a length of $30 \mathrm{~mm}$, and a wire diameter of $\varnothing=0.1 \mathrm{~mm}$. Its coarse mesh, $5-7$ tetrahedral faces per wire cross-section, contains more than half a million 4-node tetrahedral elements.
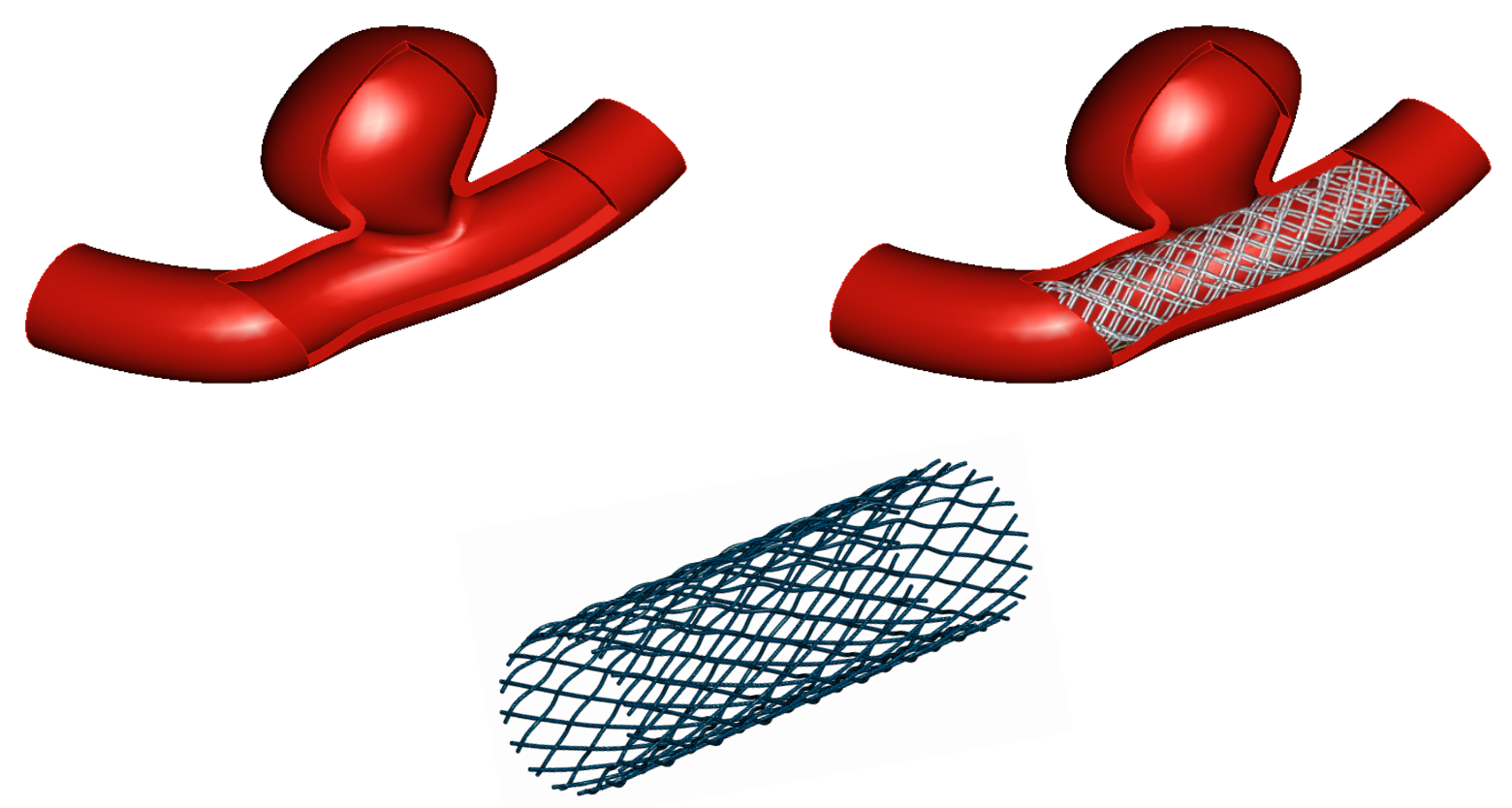

Figure 5. CAD model of a parent vessel with a side wall aneurysm (top left). Schematic illustration of a wire multi-layer stent, which reduces blood flow into the aneurysm (top right). The cutout is for visualization purpose only. A coarse finite element model of a wire two-layer stent counts $546 \mathrm{~K}$ tetrahedral elements (bottom). 
First, we discretize the unstented artery vessel, imposing a uniform node spacing for the whole medium. Tetrahedral meshes were generated by an advancing front, followed by a tetrahedral filler technique, in order to produce high-quality, quasi-uniform meshes with a low element size variance. Consecutive levels of mesh refinement are presented in fig. 6. Computation time, required to simulate one or several cardiac cycles could then be related to a spatial mesh resolution, by solving a given hemodynamic problem for each of the presented discretization. Freefem++ open source finite element code was used to compute a steady-state solution of the Stokes equations. The velocity-pressure fields were discretized by the Taylor-Hood element ((P2/P1) finite element basis). The blood was assumed to behave like an incompressible Newtonian fluid, with a constant dynamic viscosity of $3.5 \cdot 10^{-3} \mathrm{~Pa} \cdot \mathrm{s}$, and an homogeneous density of $1060 \mathrm{~kg} \cdot \mathrm{m}^{-3}$. We do not consider the compliance of arterial walls due to the complexity of the numerical modeling and requirement for a fluid structure interaction environment to solve a coupled problem. The assumption of rigid wall is based on [9], where the authors conclude that a presence of wall motion does not have significant influence of the global fluid dynamic characteristics of the femoral artery bifurcation. The inflow boundary condition is based on the constant pressure profile of $80 \mathrm{mmHg}$. We imposed the usual non-slip boundary conditions on the vessel wall, while a pressure drop of $0.07 \mathrm{mmHg}$ was prescribed on the outflow boundary. In addition, the tangential velocity component was set to be zero on the non-slip boundaries, $\mathbf{u} \cdot \tau_{\mid \Gamma_{\text {in }}, \Gamma_{\text {out }}}=0$. These boundary conditions together with a pressure gradient establish a steady laminar flow with a Reynolds number $R e=443$, and a flow rate of $11.51 \mathrm{ml} / \mathrm{sec}$.

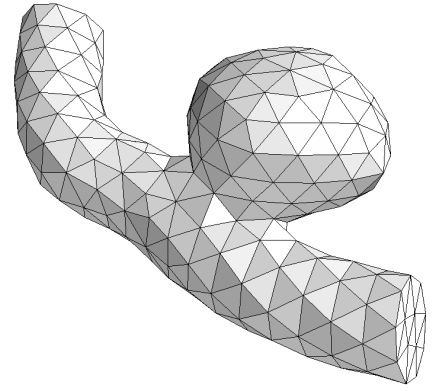

(a) $h=3.5$

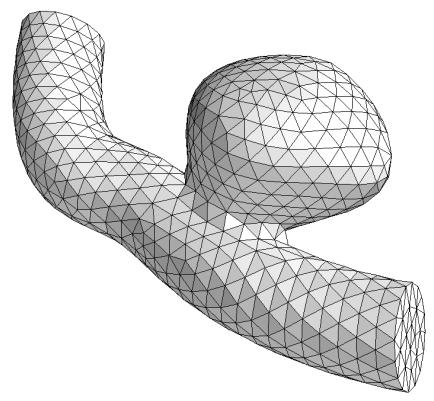

(d) $h=2.0$

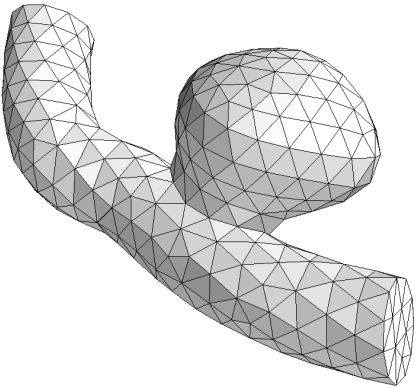

(b) $h=3.0$

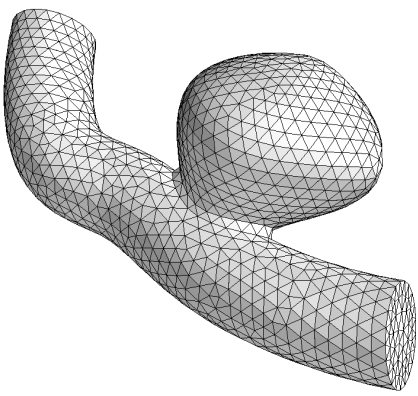

(e) $h=1.5$

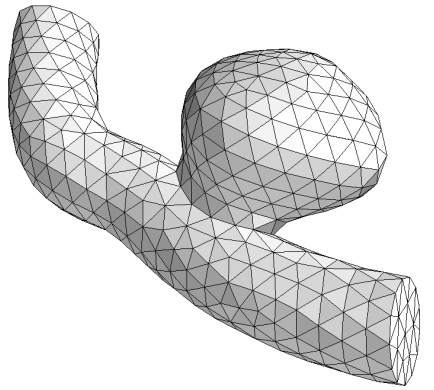

(c) $h=2.5$

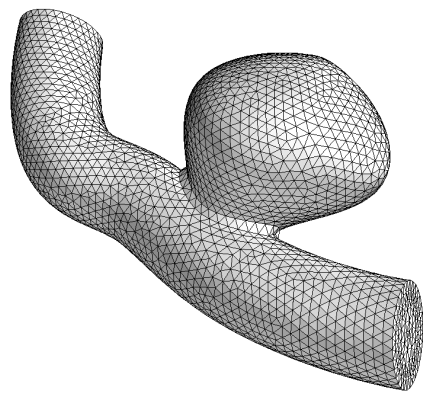

(f) $h=1.0$

FiguRE 6. Different levels of mesh refinement. Quasi-uniform meshes with a prescribed discretization element size $h$ in millimeters. An overall mesh, data are reported in table 1. 
We made use of a non-parallel version of a conjugate gradient solver with a convergence criteria of $10^{-8}$. To preserve the positive-definiteness of the global matrix, a penalization term of order $10^{-12}$ is introduced (see for instance p. 210-214 [4]). Identical results have been obtained by the GMRES iterative solver without penalization term, though it requires more memory to operate. An overall mesh, finite element, and computation data are organized in table 1. For a given discretization parameter $h$, it reports a number of produced mesh nodes, tetrahedral elements, degrees of freedom, non-zero coefficients of the symmetric finite element matrix, numerically computed flow rates in units of $\mathrm{ml} / \mathrm{sec}$. Solution time, in seconds, required to solve the stationary Stokes equations is reported in the extreme right column. The computational results reported in table 1 reveal that even the finest discretization of a fluid medium would not be sufficient to properly incorporate a coarse finite element model of the wire two-layer stent, presented in fig. 5. The finest mesh discretization parameter is 5 times larger than a wire diameter.

\begin{tabular}{|c|r|r|r|r|r|r|}
\hline$h$ & nodes & tetrahedra & dof & !=0 coef. & flow rate & cpu time \\
\hline \hline 3.5 & 443 & 1664 & 8918 & 385982 & 9.87 & 17 \\
3.0 & 685 & 2778 & 14272 & 632774 & 10.17 & 39 \\
2.5 & 1184 & 5225 & 25628 & 1166637 & 10.61 & 126 \\
2.0 & 2012 & 9278 & 44483 & 2052252 & 11.10 & 324 \\
1.5 & 4725 & 23456 & 108144 & 5103210 & 11.29 & 1363 \\
1.0 & 14276 & 75051 & 335807 & 16124234 & 11.49 & 10499 \\
0.8 & 28257 & 153582 & 675171 & 32749351 & 11.51 & 39314 \\
0.5 & 109211 & 616710 & 2657609 & 130403575 & 11.51 & 378162 \\
\hline
\end{tabular}

TABLE 1. Mesh data summary, non-zero coefficients of the symmetric finite element matrix, computed flow rate $[\mathrm{ml} / \mathrm{sec}]$, computation time [sec] of the steady-state Stokes equations.

Remark: The tasks were executed on the laboratory cluster, powered by 16 Intel Xeon E5462 @2.80GHz processors; $12 \mathrm{~Gb}$ of available RAM memory are designated for each two processors. For the finest mesh, $h=0.5,4 \mathrm{~Gb}$ of memory were allocated. Reported cpu time represents the total cpu time taken by one single processor to obtain the converged solution. We note that since each discretization was built in the stand alone way, each processor worked independently and there was no communication or synchronization overhead in the calculations. Each processor was assigned only one mesh and one variational problem to be resolved. We have repeated each computation several times, observing negligible variance in computation time.

In the second part of our numerical experiment, we simplify the original stent model, by replacing it with a pattern of unattached ring-like struts across an aneurysm throat. A similar two-dimensional version has been recently proposed in [10]. Two modeling techniques were tested to place stent wires. The first technique was to construct stent wires, completely enclosed by the blood medium. Wire centers were displaced into the parent vessel from the outer boundary by $3 / 2$ of the wire radius. An automated mesh generator had difficulties to properly define all enclosed surfaces, and to complete a meshing procedure. Moreover, this technique produced extremely small elements, located between stent wires and vessel boundary. Therefore, a unique six-wire model was constructed, fig. 7 (left). Almost worthless, it takes 214 hours to solve the Stokes equations, using a non-parallel iterative solver. A model related data was summarized in table 2 .

\begin{tabular}{|c|c|c|c|c|c|r|r|}
\hline wire $\varnothing$ & $h$ & nodes & tetrahedra & dof & $!=0$ coef. & flow rate & cpu time \\
\hline \hline 0.8 & $0.1-0.5$ & $218.9 \cdot 10^{3}$ & $1.2 \cdot 10^{6}$ & $5.3 \cdot 10^{6}$ & $261.4 \cdot 10^{6}$ & 8.34 & 769384 \\
\hline
\end{tabular}

TABLE 2. Stented aneurysm with completely enclosed stent wires: mesh data summary, nonzero coefficients of the symmetric finite element matrix, computed flow rate [ml/sec], computation time $[\mathrm{sec}]$ of the steady-state Stokes equations. 

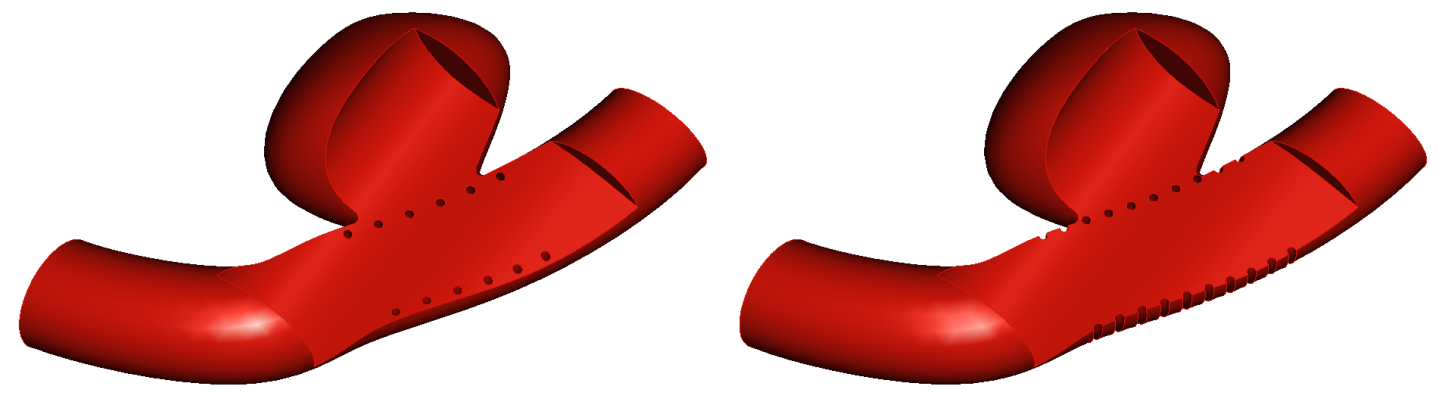

FiguRE 7. Three-dimensional CAD model of a saccular aneurysm within struts pattern across the aneurysm throat. Wire diameter is $\varnothing=0.8 \mathrm{~mm}$. The cutout exposes struts location, and is for visualization purpose only. Stents struts are completely enclosed by the blood medium (left), struts are partially displaced outside of the blood medium (right).

The second strategy was to partially enclose stent wires by the blood medium; thus, wire centers were displaced into the vessel by $1 / 6$ of the wire radius. Wires were cloned along the parent vessel direction with respect to its curvature. The distance between two wire centers is $2 \mathrm{~mm}$, fig. 7 (right). Four separate ten-wire models were constructed. Keeping the same distance between wire centers, we have consequently decreased a wire diameter, from 0.9 to $0.6 \mathrm{~mm}$. We note that it is, however, about 10 times larger than the actual wire diameter used for commercial stents. Locally refined, adaptative meshes were built using the octree algorithm, imposing a nodal spacing of $0.12 \mathrm{~mm}$ around stent struts. The transitional element distribution between the respective regions of refined and global mesh density is presented in fig. 8 .

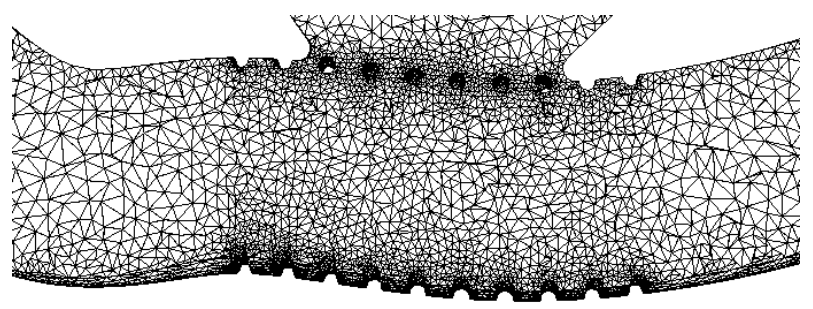

FiguRE 8. Finite element model of a saccular aneurysm within ten partially enclosed struts of $\varnothing=0.8 \mathrm{~mm}$. Zoom view over the stented region. A cross-section of the stent strut is represented by approximately 16 elements. Discretization parameter $h=0.7$ for a global domain, $h=0.12$ near the stent struts.

\begin{tabular}{|c|c|r|r|c|c|r|r|}
\hline wire $\varnothing$ & $h$ & nodes & tetrahedra & dof & !=0 coef. & flow rate & cpu time \\
\hline \hline 0.9 & $0.12-0.7$ & 95196 & 481845 & $2.2 \cdot 10^{6}$ & $104.5 \cdot 10^{6}$ & 9.90 & 235311 \\
0.8 & $0.12-0.7$ & 97405 & 495366 & $2.2 \cdot 10^{6}$ & $107.3 \cdot 10^{6}$ & 10.10 & 180206 \\
0.7 & $0.12-0.7$ & 99913 & 511385 & $2.3 \cdot 10^{6}$ & $110.6 \cdot 10^{6}$ & 10.23 & 214308 \\
0.6 & $0.12-0.7$ & 100184 & 515291 & $2.3 \cdot 10^{6}$ & $111.3 \cdot 10^{6}$ & 10.49 & 233170 \\
\hline
\end{tabular}

TABLE 3. Stented aneurysm with partially enclosed stent wires: wire diameter, mesh data summary, non-zero coefficients of the symmetric finite element matrix, computed flow rate $[\mathrm{ml} / \mathrm{sec}]$, computation time [sec] of the steady-state Stokes equations. 

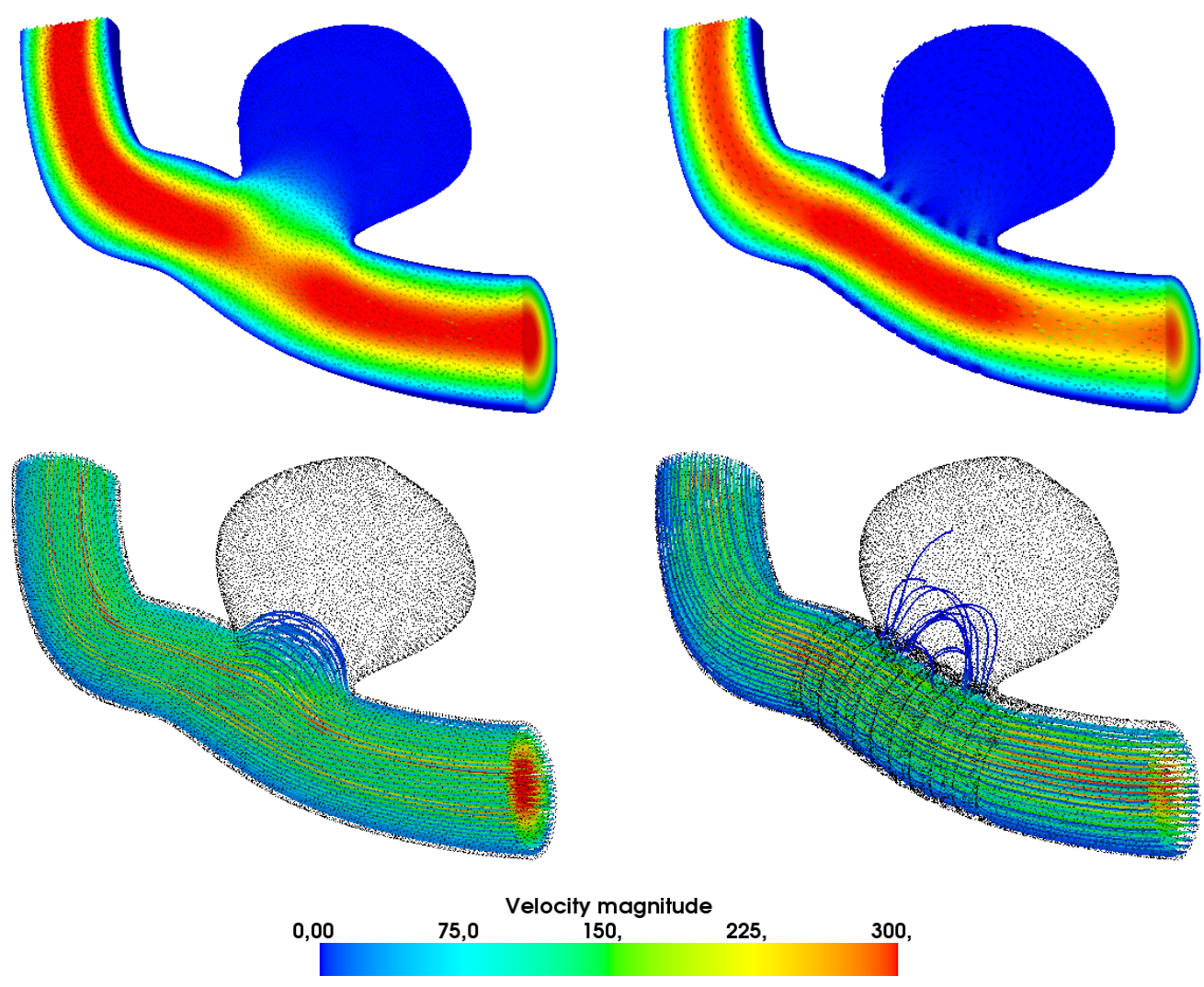

FiguRE 9. Stationary velocity field (top) and streamlines (bottom) computed before (left) and after (right) stent treatment.

The results of computations show that the presence of a stent induces a truly remarkable change of a blood flow near the throat region, fig. 9. In the case of a stented vessel the streamlines are not bent towards the aneurysm pouch, but remain similar to the bulk flow behavior. A parabolic flow profile is observed at the extreme ends of the vessel. The flow rates were computed at the upstream and downstream boundaries. The presence of the stent struts decreased the flow rate in the parent vessel. It averages the pressure inside the aneurysmal sac (this fact was already proved rigorously in [10] in 2D), and eliminates neck singularities, see fig. 10 (bottom left). Velocity vectors, depicted in fig. 11 illustrate that after stent placement, the aneurysmal vortex was no longer present. This confirms in 3D results theoretically proved in 2D in [10]. Adaptive refinement and extremely fine mesh found to be insufficient to properly model commercial multi-layer stents. It is evident that direct finite element simulations could give an additional insight, a better understanding of blood flow nature within a specific stent design, but we actually need much more computational power to simulate a pulsatile flow, where hundreds of time steps should be computed within one cardiac cycle. For this reason a work in preparation [11] aims at incorporating homogenized interface conditions and at providing some quantitative averaged results useful for clinical purposes.

\section{REFERENCES}

[1] Y. Achdou, O. Pironneau, and F. Valentin. Effective boundary conditions for laminar flows over periodic rough boundaries. $J$. Comput. Phys., 147(1):187-218, 1998.

[2] D. Bresch and V. Milisic. High order multi-scale wall laws : part i, the periodic case. accepted for publication in Quart. Appl. Math. 2008. 
[3] C. Choquet and A. Mikelić. Laplace transform approach to the rigorous upscaling of the infinite adsorption rate reactive flow under dominant Peclet number through a pore. Appl. Anal., 87(12):1373-1395, 2008.

[4] A. Ern and J.-L. Guermond. Theory and Practice of Finite Elements, volume 159 of Applied Mathematical Series. SpringerVerlag, New York, 2004.

[5] F. Hecht, O. Pironneau, A. Le Hyaric, and K. Ohtsuka. FreeFem++ (Version 2.24-3). Laboratoire Jacques-Louis Lions, Université Pierre et Marie Curie, 2008. http://www.freefem.org/ff++/ftp/freefem++doc.pdf.

[6] W. Jäger and A. Mikelic. On the effective equations for a viscous incompressible fluid flow through a filter of finite thickness. Comm. Pure Appl. Math., 1998.

[7] W. Jäger and A. Mikelić. Couette flows over a rough boundary and drag reduction. Commun. Math. Phys., 232(3):429-455, 2003.

[8] W. Jäger, A. Mikelić, and N. Neuss. Asymptotic analysis of the laminar viscous flow over a porous bed. SIAM J. Sci. Comput., 22(6):2006-2028, 2001.

[9] Y.-H. Kim, J.-E. Kim, I. Yasushi, A. M. Shih, B. Brott, and A. Anayiotos. Hemodynamic analysis of a compliant femoral artery bifurcation model using a fluid structure interaction framework. Annals of Biomedical Engineering, 36(11):1753-1763, 2008.

[10] V. Milišić. Blood-flow modelling along and trough a braided multi-layer metallic stent. submitted, preprint available at http: //hal.archives-ouvertes.fr/hal-00409446/fr/.

[11] V. Milišić. Homogenized stents for blood flow simulations in cardio-vascular junctions and aneurysms. In preparation.

[12] J. Nečas. Les méthodes directes en théorie des équations elliptiques. Masson et Cie, Éditeurs, Paris, 1967. 

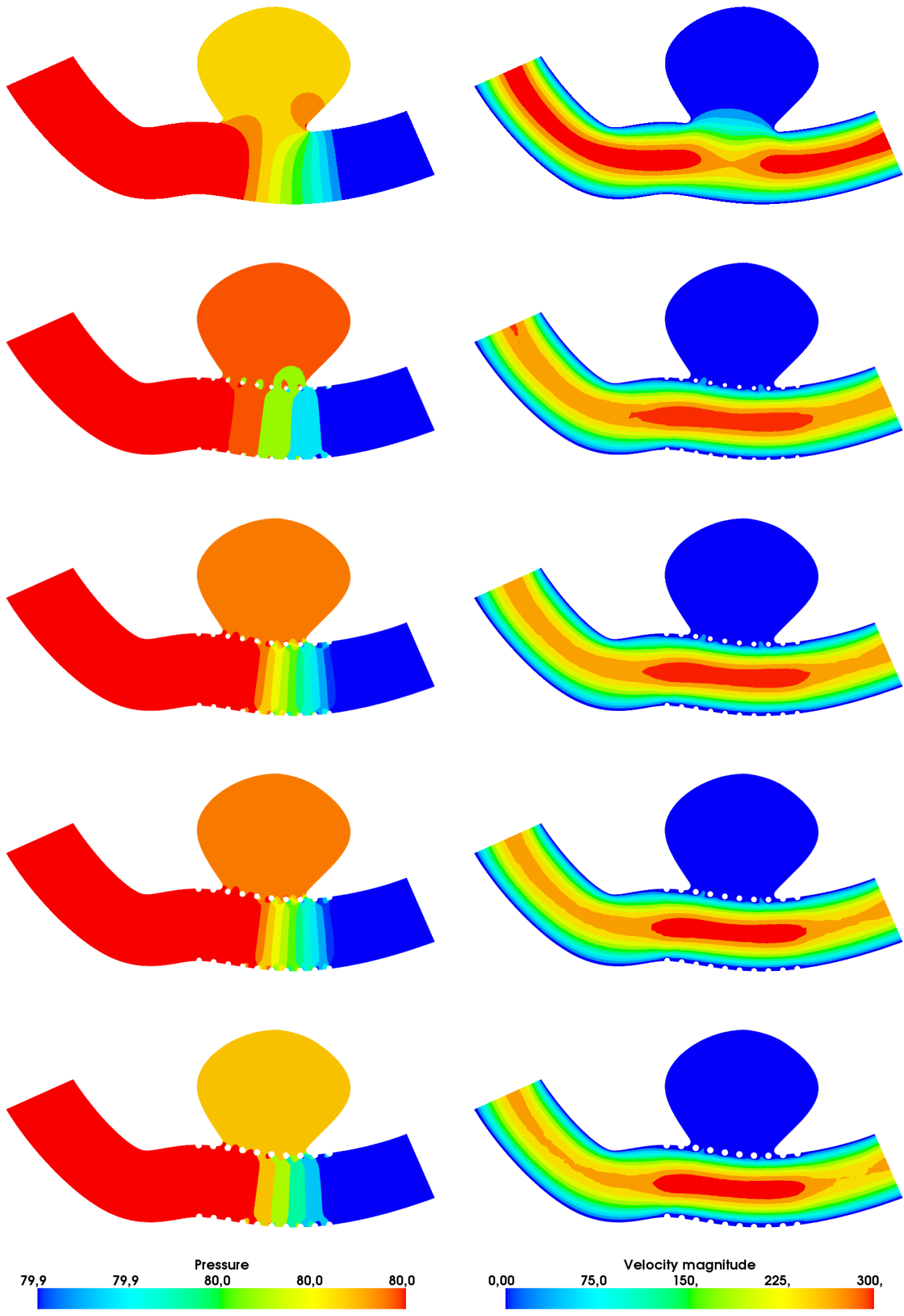

Figure 10. Sequence of pressure (left), velocity (right) solution contours. From top to bottom: unstented vessel, wire $\varnothing=0.6 \mathrm{~mm}, 0.7 \mathrm{~mm}, 0.8 \mathrm{~mm}$, and $0.9 \mathrm{~mm}$, respectively. 


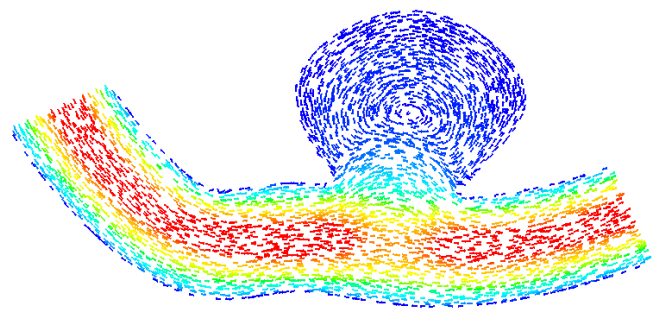

(a) no stent

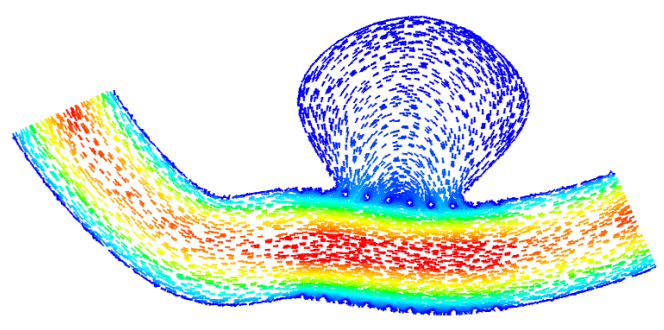

(c) $\varnothing=0.6 \mathrm{~mm}$

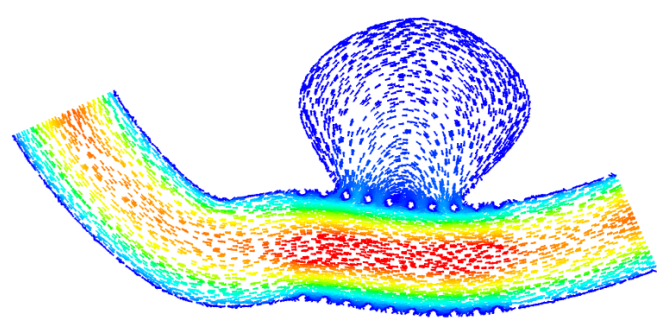

(e) $\varnothing=0.8 \mathrm{~mm}$

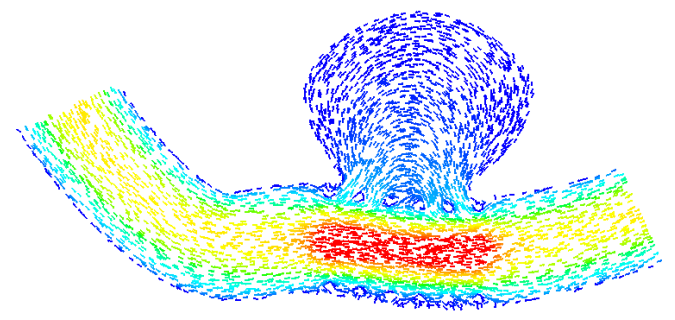

(b) $\varnothing=0.8 \mathrm{~mm}$ (completely enclosed wires)

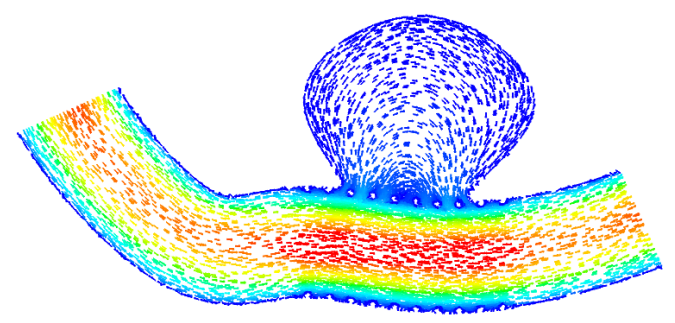

(d) $\varnothing=0.7 \mathrm{~mm}$

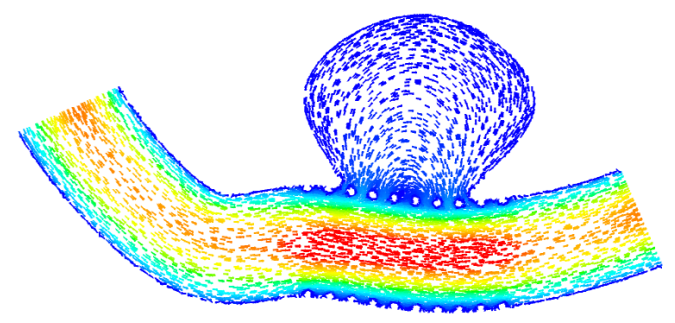

(f) $\varnothing=0.9 \mathrm{~mm}$

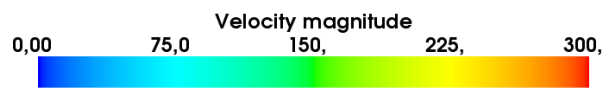

FIGURE 11. Velocity vectors colored by magnitude (not scaled arrow symbols). 